\title{
A PARTICIPAÇÃO DA FISIOTERAPIA NA RESOLUBILIDADE DA ATENÇÃO BÁSICA EM BLUMENAU - SC
}

\author{
Participation of physical therapy in primary care in solvability of \\ Blumenau - SC
}

\section{Participación de fisioterapia en la atención primaria en solvencia de Blumenau - SC}

\author{
Graziele Paula Gonçalves ${ }^{1}$ \\ José Francisco Gontan Albiero ${ }^{2}$
}

\begin{abstract}
RESUMO
A Estratégia de Saúde da Família (ESF) promove expansão e qualificação da Atenção Básica visando maior resolubilidade e a parceria com a Universidade fortalece este proposto. Esta pesquisa objetiva identificar a participação da fisioterapia nas comunidades das ESF Enfermeira Tânia Leite e Áurea Pfuetzenreiter de Blumenau - SC, na resolubilidade da Atenção Básica. Pretende conhecer os motivos dos encaminhamentos para a Fisioterapia; identificar os encaminhamentos e providências aos sujeitos encaminhados; conhecer o perfil psicosociográfico dos sujeitos encaminhados; perceber o papel do sujeito na construção e manutenção da sua saúde. É uma pesquisa de investigação científica qualitativa observacional. Os dados foram analisados através dos resultados estatísticos dos questionários e das entrevistas semi-estruturadas. Foram realizadas 87 entrevistas com sujeitos que participaram das atividades de fisioterapia do período de fevereiro a outubro de 2010. Então, construiu-se 4 grupos que melhor identificam os resultados encontrados: Grupo 1 - Promoção de Saúde; Grupo 2 - Resolubilidade atingida com ou sem limitações; Grupo 3 - Encaminhamento para outro nível de atenção; Grupo 4 - Não adesão. O G1 foi destacado 21 vezes; G2, 46; G3, 1; G4, 19. Com base nos resultados, sugere-se que a participação da fisioterapia na Atenção Básica é resolutiva e positiva para a grande maioria dos sujeitos que participaram das atividades propostas. Palavras-chave: Atenção Básica. Fisioterapia. Resolubilidade.
\end{abstract}

\begin{abstract}
The Family Health Strategy (FHS) describes the Primary Care aiming to increase the resolution, together with the University strengthens this proposal. This research aims to identify the role of physiotherapy in the communities of the FHS Enfermeira Tania Leite and Aurea Pfuetzenreiter Blumenau - SC, the solvability of Primary Care. Furthermore, knowing the reasons of referral, to identify referrals and arrangements sent to the subjects; know psicosociográfico profile of the subjects referred; understand the role of subject in the construction and maintenance of their health. It is a qualitative survey of scientific observation. Data were analyzed by statistical results of questionnaires and semi-structured interviews. We conducted 87 interviews with people who participated in the activities of physical therapy from February to October 2010. Then we constructed four groups that best represent the results: Group 1 - Health Promotion, Group 2 - solving achieved with or without limitations, Group 3 - Referral to another level of attention and Group 4 - Nonadherence. G1 was deployed 21 times, G2 46, G3, 1; G4, 19. Thus, it is suggested that the participation

\footnotetext{
${ }_{1}^{1}$ Graduanda do $9^{\circ}$ semestre de Fisioterapia da Universidade Regional de Blumenau; Bolsista do programa de pesquisa PIPe/Artigo 170 2010/2011. Centro de Ciências da Saúde - Departamento de Fisioterapia, Universidade Regional de Blumenau. Rua Edmundo Hort, n¹84, Blumenau (SC), CEP: 89026-410. Telefone: (47) 99842272, e-mail: graziipg@hotmail.com.

${ }^{2}$ Prof. Me da Universidade Regional de Blumenau; Orientador do programa de pesquisa do PIPe/Artigo 170 2010/2011.

${ }^{3}$ Esta pesquisa foi financiada pelo PIPe/Artigo 170 2010/2011.
} 
of physiotherapy in primary care is to solving the vast majority of subjects who participated in the activities proposed.

Keywords: Primary care. Physiotherapy. Solvability.

\section{RESUMEN}

La Estrategia de Salud de la Familia (ESF) describe la Atención Primaria con objetivo de aumentar la resolución, junto con la Universidad refuerza esta propuesta. Esta investigación tiene como objetivo identificar el papel de la fisioterapia en las comunidades de la leche ESF Enfermeira Tania Leite y Aurea Pfuetzenreiter Blumenau - SC, la solvencia de la Atención Primaria. Por otra parte, saber las razones de la remisión, para identificar las referencias y los acuerdos enviados a los sujetos, conocer el perfil psicosociográfico de los temas tratados; entender el papel del sujeto en la construcción y el mantenimiento de su salud. Se trata de un estudio cualitativo de la observación científica. Los datos fueron analizados por los resultados estadísticos de los cuestionarios y entrevistas semi-estructuradas. Hemos llevado a cabo 87 entrevistas con personas que participaron las actividades de terapia física, de febrero a octubre de 2010. Entonces construimos cuatro grupos que mejor representan los resultados: Grupo 1 - Promoción de la Salud, Grupo 2 la solución de lograr con o sin limitaciones, el Grupo 3 - Referencia a otro nivel de atención y Grupo 4 - Falta de adhesión. G1 se desplegó en 21 ocasiones, 46 G2, G3, 1; G4 de 19. Por lo tanto, se sugiere que la participación de la fisioterapia en atención primaria es resolver la gran mayoría de los sujetos que participaron en las actividades propuestas.

Palabras clave: Atención primaria. Fisioterapia. Solvencia.

\section{Introdução}

A criação do SUS sugeriu em prol da integralidade e da resolubilidade do primeiro nível da atenção, novos processos de trabalho que integrem ações de promoção da saúde, prevenção de doenças, cura e reabilitação (REZENDE, 2007). Desta forma, o Ministério da Saúde prioriza em seus projetos políticos, a organização da ESF para promover expansão e qualificação da atenção básica. Seu desafio é ampliar as fronteiras de atuação focando uma maior resolubilidade da atenção, onde a Saúde da Família é compreendida como a estratégia principal para mudança deste modelo (BRASIL, 2006).

O curso de Fisioterapia da Universidade Regional de Blumenau - FURB, prepara o profissional para proporcionar bem-estar aos pacientes, recuperar as capacidades funcionais, promover a prevenção global e adaptar o indivíduo ao meio (FURB, 2009). Desenvolve atividades estágio curricular obrigatório na área de Saúde Coletiva, em parceria com a Secretaria Municipal de Saúde de Blumenau (SEMUS), desde 2001.

Com isso, esta pesquisa objetivou identificar a participação da fisioterapia nas comunidades da ESF Enfermeira Tânia Leite e Áurea Pfuetzenreiter de Blumenau - SC, na resolubilidade da Atenção Básica, assim como, conhecer os motivos dos encaminhamentos da unidade e comunidade para o setor de Fisioterapia, identificar os encaminhamentos e providências aos sujeitos encaminhados, conhecer o perfil psicosociográfico dos sujeitos encaminhados $e$ perceber o papel do sujeito na construção $e$ manutenção da sua saúde. E, justifica-se, para perceber o papel, a função e a contribuição da fisioterapia na Atenção Básica.

\section{Materiais e métodos}

Foi realizada uma pesquisa de investigação científica qualitativa observacional. Os sujeitos da pesquisa foram todos os usuários que foram encaminhados e avaliados na fisioterapia no período de fevereiro a outubro de 2010, nas comunidades das ESFs Enfermeira Tânia Leite e Áurea Pfuetzenreiter, em Blumenau. 
A pesquisa foi realizada com um instrumento divido em duas etapas: a primeira foi através do questionário com dados do perfil psicosociográfico dos sujeitos; e a segunda, pelo roteiro da entrevista semi-estruturada.

Para a análise dos questionários, foi utilizada estatística descritiva, agrupada em dados intervalares objetivando identificar os atendimentos, encaminhamentos e providências para os usuários.

E, as entrevistas semi-estruturadas, foram transcritas sob forma de narrativa em $1^{\text {a }}$ pessoa e, partir de então, destacou-se as frases/ sentidos principais, de acordo com a recorrência, objetivando conhecer melhor o motivo do encaminhamento para a fisioterapia, quando iniciou as atividades e a co-responsabilidade dos sujeitos com a sua saúde.

Para o melhor desenvolver da entrevista e para não desperdiçar relatos importantes, foi utilizado um gravador da marca Panasonic, modelo RR - US 450. Foi utilizado também, uma câmera fotográfica digital da marca Cássio, modelo EXILIN EX - Z75, para registrar os momentos mais marcantes da pesquisa e um pen drive da marca Kingston, modelo DataTraveler, 4GB.

Após a aprovação do projeto no Conselho de Ética, protocolo 165/09, aplicou-se o questionário e a entrevista semi-estruturada com a sujeitos da pesquisa, juntamente com o Termo de Consentimento Livre e Esclarecido, a fim de se obter os dados relativos à pesquisa através da análise dos resultados.

\section{Análise e discussão dos resultados}

\section{Contextualização da área}

As duas ESF têm realidades locais distintas, com comunidades adaptadas ao seu meio. Os sujeitos são encaminhados para a fisioterapia pela equipe de saúde da família da unidade, escolas, creche e pela própria comunidade. Realiza-se com equipe, a análise de seus prontuários para saber seu histórico de saúde e condição social, e a partir de então, são encaminhados para avaliação fisioterapêutica, a qual reconhece suas necessidades.

Os usuários são encaminhados devido um universo amplo de objetivos, necessidades e queixas, como a prevenção e a promoção da saúde, a reabilitação, a manutenção da saúde, e consequentemente, a melhora da qualidade de vida de acordo com a visão de cada usuário.

Analisando os motivos dos encaminhados por faixa etária, de forma geral, pode-se considerar que as crianças são encaminhadas por queixas respiratórias (ex: acúmulo de secreções em vias aéreas superiores), posturais (ex: estirões de crescimento) e neurológicas (ex: atraso de desenvolvimento motor); os idosos, por problemas reumatológicos (ex: osteoartrose), neurológicos (ex: Acidente Vascular Encefálico) e por promoção e prevenção da saúde; os adultos, no caso das mulheres por período gestacional, por promoção e prevenção da saúde, e por Distúrbios Osteomusculares Relacionados ao Trabalho (DORT), que de acordo com Garcia et al (2004), são, atualmente, um dos principais problemas de saúde pública e os responsáveis por quase $90 \%$ dos afastamentos do trabalho. Em todos os grupos etários pode haver outros motivos de encaminhamentos, porém esses foram identificados com maior freqüência.

As condutas fisioterapêuticas realizadas são de acordo com necessidade real de cada indivíduo, desta forma, as modalidades fisioterapêuticas se caracterizam em atendimentos domiciliares usando os princípios da equidade, atendimentos individuais, grupos terapêuticos, visitas de orientação e referência para outros níveis de saúde quando necessário. Vale ressaltar que as atividades fisioterapêuticas exercidas em grupo, não são condicionantes a estar vinculada a consulta medica ou a distribuição de 
medicamentos e receitas após a participação das atividades.

\section{Contextualização dos sujeitos}

A população foi composta por 87 sujeitos, dentre eles $78,16 \%$ são mulheres $e$ $21,84 \%$ são homens. Figueiredo (2005) expõe que as Unidades Básicas de Saúde (UBS) são compostas pela maioria de usuários do gênero feminino, crianças e idosos.

De acordo com a faixa etária, 8,04\% são crianças, $41,39 \%$ são adultos e $50,57 \%$ são idosos. Segundo Lima-Costa et al (2003), uma das consequências do crescimento da população idosa é o aumento da demanda por serviços de saúde e sociais nesta classe.

Os dados do perfil dos indivíduos colhidos no questionário identificam que em relação à escolaridade 10,34\% são analfabetos, $72,42 \%$ fizeram somente o ensino fundamental e 17,24\% completaram o ensino médio, não tendo nenhum sujeito com graduação em ensino superior. Meireles (2007) afirma que o baixo nível de escolaridade deve ser considerado no planejamento de programas e ações, tanto no nível coletivo quanto no individual. O nível educacional interfere diretamente no desenvolvimento da consciência sanitária, na capacidade de entendimento do tratamento prescrito e na prática do auto-cuidado, interferindo diretamente na relação equipe de saúde/paciente.

À respeito da profissão dos sujeitos, $6,89 \%$ dizem ser estudantes, $19,55 \%$ desempregados, $14,94 \%$ empregados, $1,15 \%$ trabalhadores sem registro, 1,15\% autônomo e 56,32\% aposentados. Esse valor demonstra que a maior parte dos usuários são aposentados e que tem maior tempo livre para poderem participar das atividades da fisioterapia.

Considerando o motivo, ou a queixa principal que levou o usuário a participar das atividades da fisioterapia $27,58 \%$ dos sujeitos vieram apenas em busca da promoção e da prevenção à saúde, demonstrando o reconhe- cimento da população em prevenir e promover saúde. Entretanto a busca pela manutenção e reabilitação através de queixas já instaladas ainda são relativamente maiores, sendo que 50,57\% vieram devido a queixas músculo-esqueléticas, $19,09 \%$ por queixas neurológicas, 2,29\% por queixas cardiorrespiratórias e $3,44 \%$ por outros tipos de queixas.

Os dados sob os hábitos de vida demonstram que $47,12 \%$ são sedentários e $52,88 \%$ praticam alguma atividade física ao menos uma vez durante a semana. Mcardle et al (2008) afirmam que o sedentarismo é fator de risco para diversas doenças.

Com relação aos indivíduos que já fizeram fisioterapia, os resultados demonstram que $40,22 \%$ dos sujeitos entrevistados já tiveram contato alguma vez com a fisioterapia, embora, a grande maioria, foi através do nível secundário e terciário de atenção à saúde. Ribeiro (2002) afirma que inserção do fisioterapeuta nos serviços de atenção primária à saúde é um processo em construção, devido à visão de um modelo antigo em que o fisioterapeuta é reconhecido como reabilitador.

De acordo com o perfil da amostra, $34,48 \%$ dos indivíduos tem Diabetes, $51,72 \%$ são hipertensos e $36,78 \%$ fazem uso contínuo de medicação para controlar suas doenças, estando presentes mensalmente na unidade de saúde em busca de seus medicamentos.

A Hipertensão Arterial e o Diabetes Mellitus constituem os principais fatores de risco populacional para as doenças cardiovasculares e são agravos de saúde pública dos quais até $80 \%$ dos casos podem ser tratados na Atenção Básica (Brasil, 2001). A prevenção de sequelas constitui em um dos pontos fundamentais da atenção ao doente crônico, visto que elas podem resultar em incapacidades funcionais permanentes (MARCON et al, 2004).

Ainda em relação ao perfil da amostra, os dados apontam que $18,39 \%$ dos sujeitos são membros ativos na comunidade, ou seja, 
participam ativamente das questões políticas e sociais que envolvem suas comunidades como: reuniões do Conselho Local de Saúde; reuniões da Associação de Moradores, atividades oferecidas pelas igrejas e creches; demonstrando suas corresponsabilidades em lutar para fazer e trazer melhorias para si e para sua comunidade.

\section{Grupos de acordo com a resolubilidade}

O Grupo 1 (G1), descreve as pessoas que participaram das atividades de fisioterapia nas ESF apenas por vontade própria, sem ter alguma queixa, visando a promoção e a prevenção da saúde.

Grupo 2 (G2), os indivíduos que participaram da fisioterapia vieram devido alguma queixa principal e apresentaram um quadro positivo de melhora, em suas dificuldades e em sua dependência e/ou independência nas atividades de vida diária (AVD'S) e atividades instrumentais de vida diária (AIVD'S).

No Grupo 3 (G3), os indivíduos que foram avaliados na fisioterapia e foram encaminhados para outros serviços, visto que o tratamento fisioterapêutico disponível na Atenção Básica não supre suas necessidades.

O Grupo (G4), corresponde às pessoas que não aderiram aos serviços oferecidos pela fisioterapia da FURB, independente do motivo.

Observa-se na tabela, os resultados da classificação dos encaminhamentos dos sujeitos que participaram das atividades de fisioterapia nas duas ESF.

\begin{tabular}{|l|l|l|}
\hline Classificação & $\begin{array}{l}\text { Usuários } \\
(\mathbf{8 7 )}\end{array}$ & $\begin{array}{l}\text { Porcenta- } \\
\text { gem (100\%) }\end{array}$ \\
\hline $\begin{array}{l}\text { G1 - Promoção da Saúde } \\
\text { G2 - Resolutivo conforme a } \\
\text { queixa principal }\end{array}$ & $\mathbf{2 1}$ & $52,14 \%$ \\
\hline $\begin{array}{l}\text { G3 - Encaminhado para } \\
\text { outro serviço de atenção } \\
\text { secundária ou terciária }\end{array}$ & 1 & $1,15 \%$ \\
\hline G4 - Não aderiu & 19 & $21,83 \%$ \\
\hline TOTAL & $\mathbf{8 7}$ & $\mathbf{1 0 0 \%}$ \\
\hline
\end{tabular}

TABELA 1 - RESULTADOS DA CLASSIFICAÇÃO DOS INDIVÍDUOS ENCAMINHADOS PARA A FISIOTERAPIA

\section{Promoção da saúde (G1)}

Com base nos resultados obtidos demonstrados na tabela, observa-se que o grupo 1 (G1), referente as pessoas que participam das atividades da fisioterapia visando a promoção da saúde, foi encontrado em $24,14 \%$ dos indivíduos. Esses resultados demonstram que o trabalho proposto pelo Ministério da Saúde para a Atenção Básica, está se fortalecendo através da inserção dos acadêmicos de fisioterapia da FURB, nas ESF Enfermeira Tânia e Leite e Áurea Pfuetzenreiter de Blumenau, conforme exposto nas falas abaixo dos sujeitos entrevistados:

"Eu gosto de vim aqui fazer exercícios porque sei que é bom para minha saúde e eu não quero ficar doente (sujeito 73)."

Pode-se identificar que a população está compreendendo a importância de promover e prevenir saúde, e assim, torna-se efetiva a co-responsabilidade dos indivíduos perante a construção e manutenção da sua saúde, visando o bem-estar e a qualidade de vida.

"Eu participo porque quero cuidar da minha saúde e me manter sempre bem. Eu adoro participar e me sinto muito disposta ao longo do dia (sujeito 68)."

"Eu participo das atividades da fisioterapia aqui porque eu quero me cuidar e ter uma boa saúde. Eu sou diabética é muito importante para mim fazer atividade física e cuidar da minha saúde (sujeito 25)".

Segundo a OMS in Fleck (2000), qualidade de vida é "a percepção do indivíduo acerca da posição de vida, de acordo com o contexto cultural e os sistemas de valores nos quais vive e em relação aos seus objetivos, expectativas, padrões e preocupações".

Outro aspecto importante a salientar referente aos resultados encontrados neste grupo é mudança de concepção da atuação fisioterapêutica voltada para a reabilitação, agora é desfrutada pela comunidade através da atenção básica à saúde. Sabe-se hoje, que o fisioterapeuta tem capacidade de desenvolver atividades em 
todos os níveis de atenção à saúde, de primário à terciário, dentro da equipe interdisciplinar (BRASIL et al, 2005).

\section{Resolutivo conforme a queixa principal (G2)}

O grupo 2 (G2), referente aos indivíduos que vieram à fisioterapia devido alguma queixa principal e apresentaram um quadro positivo de melhora, destacou-se em 52,88\% entrevistados. Isso demonstra que a fisioterapia está sendo resolutiva e se mostra eficaz na Atenção Básica através de ações que visam a prevenção, a promoção e a reabilitação à saúde dos indivíduos.

"Depois que comecei a fazer fisioterapia aqui eu fiquei bem melhor, a dor que tenho por causa da artrose diminuiu muito (sujeito 48)".

Atenção Básica à Saúde é um dos pilares da organização de sistemas de saúde devido seu grande potencial de resolver parte significativa das queixas dos usuários (WORLD HEALTH ORGANIZATION, 2008).

"Desde o acidente eu faço fisioterapia em casa e melhorei bastante. Hoje eu até consigo andar um pouco com auxílio do andador (sujeito 70)".

Segundo Rodrigues (2008), a reabilitação visa à inserção de indivíduos, no âmbito de suas vidas, organizados em um cotidiano que os remeta ao bem-estar, saúde e qualidade de vida.

Com base nos resultados encontrados neste grupo, pode-se salientar que eles se deram devido alguns fatores positivos construtores destes resultados, tais como:

- A efetividade de um trabalho fisioterapeutico: Para isso, o profissional deve olhar o sujeito de forma integral, e também, estar sensível às necessidades e às circunstâncias de vida das famílias envolvidas e dos colegas de trabalho, visando o trabalho interdisciplinaridade quando necessário. (SILVA e ROS, 2007).

- A orientação de medidas preventivas para os indivíduos darem continuidade em seu tratamento ao longo da semana em sua residência, visto que, nas duas ESF, os acadêmicos estão presentes apenas uma vez durante a semana $e$ por apenas um período.

Bensen et al (2007), afirmam que ESF tem papel central uma prática educativa voltada para a Promoção da Saúde.

"Eles me ensinam o que eu devo fazer e com isso dou continuidade ao tratamento durante a semana (sujeito 6)".

A diminuição de demanda em outros serviços, como os de atenção secundária ou terciária, proporciona redução de gastos públicos conforme exposto por Costa et al (2009): a inserção do fisioterapeuta em uma unidade de saúde previne o aumento do volume e da complexidade da atenção em saúde, reduzindo os gastos públicos.

"Eu fiz fisioterapia em uma clínica por 4 meses e melhorei bastante. Hoje eu faço somente aqui e continuei a melhorar (sujeito 54)".

Além da economia do governo, deve-se salientar a facilidade e a comodidade oferecida para os pacientes em ter a fisioterapia em sua comunidade, diminuindo assim os transtornos com o deslocamento para outro lugar que ofereça algum serviço de fisioterapia. Este fato segue o que descreve o Plano Diretor de Regionalização (PDR) proposto na NOAS 2002, elaborado na perspectiva de garantir acesso aos cidadãos, o mais próximo possível de sua residência:

"Era muito complicado para nós termos que levá-la para a clínica, e eu tinha que tirar ela do carro e colocar e eu já não tinha mais força, estava muito pesado. Agora eles vêm atender ela aqui em casa e é ótimo (sujeito 6)".

Encaminhamento para outro serviço de Atenção Secundária ou Terciária (G3)

O grupo 3 (G3), menciona os sujeitos que participaram das atividades fisioterapêuticas, mas a atenção primária não supriu a queixa principal, necessitando referência para outro serviço de atenção secundária ou terciária. 
“... fui encaminhado para a clínica de fisioterapia da porque eles falaram que eu tenho um problema de cervicobraquialgia irradiada e que eu tenho que ir para lá para resolver meu problema (sujeito 79)".

Em situações álgicas agudas é necessário técnicas de analgesia de forma intensa $e$ diária. As unidades secundárias e terciárias de atenção à saúde estão aptas a atender casos de maior complexidade (MARTINEZ, 2004).

Esta categoria foi a de menor incidência perante as demais e esse fato corresponde um resultado positivo para a resolubilidade da fisioterapia na Atenção Básica, pois dentre os dados coletados, apenas $1,15 \%$ dos indivíduos necessitou referência para outro nível de atenção.

Desta forma, pode-se considerar eficiente a fisioterapia na Atenção Básica por suprir grande parte da demanda, através de atividades de promoção, prevenção e reabilitação da saúde, visando à atenção integral da saúde dos sujeitos.

A integralidade é um conceito que permite uma identificação dos sujeitos como totalidades, ainda que não sejam alcançáveis em sua plenitude, considerando todas as dimensões possíveis que se pode intervir, pelo acesso permitido por eles próprios. É um dispositivo jurídico institucional a partir do objetivo de assegurar aos "indivíduos a atenção à saúde, dos níveis mais simples aos mais complexos, da atenção curativa à preventiva, bem como a compreensão, em sua totalidade, dos indivíduos e/ou coletividades em suas singularidades" (PINHEIRO e MATTOS, 2004).

Não aderiu (G4)

O grupo 4 (G4), que descreve os indivíduos que não aderiram aos serviços oferecidos pela fisioterapia da FURB nas ESF, destacou-se em $21,83 \%$ dos sujeitos entrevistados.

Este resultado, diz respeito aos indivíduos que marcaram avaliação fisioterapêutica $e$ não compareceram, e também, aos que fizeram avaliação, começaram o programa fisioterapêutico proposto, entretanto não deram continuidade:

"Não participei mais da fisioterapia porque é no meu horário de trabalho (sujeito 61)”.

O crescimento industrial acelerado, decorrência natural do sistema capitalista, preocupa-se mais com os lucros e deixa para segundo plano a saúde do trabalhador (PAGLIOSA, 1999).

Outras situações que aparecem foram de indivíduos que não conhecem o trabalho fisioterapêutico:

"Eu não participei da fisioterapia porque não sei o que é (sujeito 56)".

Considerando-se que a Fisioterapia é uma profissão relativamente nova e que seu acesso ainda é limitado a pequena parte da população, o conhecimento da sua atuação, ainda hoje, é restrito (RIBEIRO, 2005).

Alguns indivíduos não puderam comparecer na fisioterapia por alguns problemas pessoais como múltiplas tarefas; cuidar de crianças; jornada dupla de trabalho; dificuldades de acesso; dificultando sua participação nas atividades da unidade. Essas situações podem ser compreendidas por determinantes sociais em saúde, segundo Marchiori e Pellegrini (2007), a situação de saúde dos indivíduos e da população, estão relacionadas com suas condições de vida e de trabalho.

"Eu não participei mais da fisioterapia porque tive que fazer uma cirurgia, mas agora eu quero continuar (sujeito 64)".

"Eu estava muito atarefada no dia que era para eu ir e acabei nem indo, depois eu acabei esquecendo (sujeito 86)".

Muitos sujeitos pararam de frequentar a fisioterapia por fazerem suas respectivas altas fisioterapêuticas. O verdadeiro profissional de saúde é aquele que respeita e valoriza a autonomia dos sujeitos sobre a sua história de vida. Autonomia para Freire (2009), significa respeito a cultura, conhecimentos empíricos, decisões e 
a capacidade do indivíduo ser reflexivo a suas práticas. Sendo assim, decidir por encerrar suas atividades em um grupo ou ainda por se julgar bem, é rotina no cotidiano das unidades.

"Não fui mais para a fisioterapia porque eu achava que não precisa mais (sujeito 65)".

Em alguns casos, pode-se perceber o empoderamento e a co-responsabilidade dos indivíduos perante suas vidas, através educação popular que a fisioterapia exerceu nas ESF. Segundo Vasconcelos (2004), a educação popular busca trabalhar pedagogicamente o homem e os grupos envolvidos no processo de participação popular, fomentando formas coletivas de aprendizado e investigação, de modo a promover o crescimento da capacidade de análise crítica sobre a realidade e o aperfeiçoamento das estratégias de luta e enfrentamento. É uma estratégia de construção da participação popular no redirecionamento da vida social.

Dessa forma, alguns sujeitos que tinham dificuldades de se deslocar até a unidade de saúde não compareceram mais, pois deram continuidade ao seu tratamento preventivo em sua residência:

"Eu não participei mais da fisioterapia porque eu faço em casa o que eles me ensinaram e não preciso mais ir até o postinho (sujeito 66)".

A educação é o principal fator contribuinte para fazer com que as pessoas tomem consciência de si e reflitam sobre suas ações para que possam lidar melhor suas decisões (FREIRE, 2002).

\section{Considerações finais}

De acordo com os resultados da presente pesquisa, a fisioterapia participou positivamente na resolução de $77,02 \%$ dos encaminhamentos dos indivíduos entrevistados que participaram da pesquisa. Este resultado diz respeito aos sujeitos classificados entre as cate- gorias de Promoção da Saúde e de Resolução conforme a queixa principal.

Os motivos dos encaminhamentos para a fisioterapia foram a promoção de saúde em $27,58 \%$ dos dados coletados, e a queixas referentes a questões musculoesqueléticas cardiorrespiratórias, neurológicas e outras, em 72,42\% dos casos. E de todos os encaminhamentos, $77,02 \%$ foram absorvidos na própria unidade.

Em relação ao perfil psicosociográfico dos sujeitos, os resultados demonstram que a maior demanda aos serviços de fisioterapia são: de acordo com o gênero, $78,16 \%$ são mulheres; em relação a faixa etária, $50,57 \%$ são idosos; à escolaridade, 70,42\% fizeram apenas o ensino fundamental; à respeito do motivo, $72,42 \%$ vieram em busca da fisioterapia por já ter alguma queixa principal; sobre os hábitos de vida, 52,88\% praticam alguma atividade física; aos que já fizeram fisioterapia, 40,22\% já precisaram dos serviços fisioterápicos ao menos uma vez; em relação as doenças crônicas, $34,48 \%$ tem Diabetes, $51,72 \%$ são hipertensos e $36,78 \%$ fazem uso contínuo de medicação para controlar suas doenças; e aos membros ativos na comunidade, $18,39 \%$ participam ativamente das questões políticas e sociais que envolvem suas comunidades.

Os dados dessa pesquisa sugerem que os sujeitos integrantes do grupo G1 $(24,14 \%)$, assumem a responsabilidade sobre os cuidadose manutenção da saúde, bem como, valorizam sua qualidade de vida, pois participam efetivamente de atividades preventivas, com objetivo principal de cuidar da saúde. E também, apontam que os sujeitos integrantes do grupo G2 (52,88\%) apresentam constância $e$ frequência nas atividades propostas, mantendo-se aderidos e dando continuidade aos cuidados em seus domicílios e ambiente de trabalho.

O fisioterapeuta presente dentro da unidade, assim como os médicos, enfermeiros e dentistas, contribui para o fortalecimento do trabalho preventivo que constitui uma ESF, 
exercendo ações de atenção primária à saúde para prevenir futuros problemas e manter um bom estado funcional do corpo, assim como a manutenção e reabilitação da saúde na medida do possível.

Desta forma, pode-se perceber que ampliação de profissionais de várias áreas de atuação na Equipe de Saúde da Família, dentre eles, o fisioterapeuta, contribui para a resolubilidade da Atenção Básica e melhoria do serviço oferecido.

\section{REFERÊNCIAS}

BENSEN, C. B. et al. A Estratégia de Saúde da Família como objeto de educação em saúde. Saúde e Sociedade, São Paulo, v. 16, n.1, jan./abr. 2007.

BRASIL. Ministério da Saúde. Política Nacional de Atenção Básica. Pactos pela Saúde. vol 4. Brasília, 2006.

BRASIL, Ministério da Saúde. Departamento de Atenção Básica. Guia prático do Programa de Saúde da Família. Brasília, DF, 2001.

BRASIL, et al. O papel do fisioterapeuta no Programa de Saúde da Família do município de Sobral - Ceará. Revista Brasileira de Promoção da Saúde, v. 1, n. 1, 2005.

COSTA, D.; FREITAS, C. M. Promoção da Saúde: conceitos, reflexões, tendências. Rio de Janeiro: FIOCRUZ, 2003. 174 p.

FIGUEIREDO, W. Assistência à saúde dos homens: um desafio para os serviços de atenção primária. Revista Ciência e Saúde coletiva, Rio de Janeiro, v. 10, n. 1, jan./ mar. 2005

FLECK, M. P. A. O instrumento de avaliação de qualidade de vida da Organização Mundial da Saúde (WHOQOL-100): características e perspectivas. Revista Ciência e Saúde Coletiva, v. 5, n. 1, p. 33-38, 2000.

FREIRE, P. Pedagogia do oprimido. Educação como pratica da liberdade. 26 ed. Rio de Janeiro: Paz e Terra, 2002.

Pedagogia da autonomia: saberes necessários à prática educativa. 39 ed. São Paulo: Paz e Terra, 2009.

FURB, Universidade Regional de Blumenau. Pró Reitoria de Pesquisa e Extensão: a organização dos cursos. Blumenau, 2009.

GARCIA, V. M. D. Análise do perfil do paciente portador de Doença Osteomuscular Relacionada ao Trabalho
(DORT) e usuário do serviço de saúde do trabalhador do SUS em Belo Horizonte. Revista Brasileira de Fisioterapia, v. 8, n. 3, p. 273-278, São Carlos, 2004.

LIMA-COSTA, M. F. et al. Desigualdade social e saúde entre idosos brasileiros: um estudo baseado na pesquisa nacional por amostra de domicílios. Cadernos de Saúde Pública, Rio de Janeiro, v. 19, n. 3, p. 745-757, 2003.

MARCHIORI, P. B.; PELLEGRINI, A. F. A saúde e seus determinantes sociais. Physis, Rio de Janeiro, v. 17, n. 1, jan./abr. 2007.

MARCON, S. S. et al. Características da doença crônica em famílias residentes na região norte de Maringá-PR: uma primeira aproximação. Acta Scientiarun, Maringá, v. 26, n. 1, p. 83-93, 2004.

MARTINEZ, J. E. et al. Perfil clínico e demográfico dos pacientes com dor músculo-esquelética crônica acompanhados nos três níveis de atendimento de saúde de Sorocaba. Revista. Acta Fisiatrica, v. 11, n. 2, p. 67-71, São Paulo, 2004.

MCARDLE, W. D.; KATCH, F.; KATCH, V. L. Fisiologia do exercício: energia, nutrição e desempenho humano. 6 ed. Rio de Janeiro: Guanabara Koogan, 2008.

MEIRELES, V. C. et al. Características dos idosos em área de abrangência do programa saúde da família na região noroeste do Paraná: contribuições para a gestão do cuidado em enfermagem. Revista Saúde e Sociedade, v. 16, n. 1, p. 69-80, jan./abr. São Paulo, 2007.

PAGLIOSA, K .M. Ruído: relação entre saúde, trabalho e qualidade de vida. 1999. Monografia de Conclusão de Curso - Centro de Especialização em Fonoaudiologia Clínica Audiologia Clínica, Porto Alegre.

PINHEIRO, R.; MATTOS, R. A. Cuidado: as fronteiras da integralidade. Rio de Janeiro: Hucitec: ABRASCO, 2004. 319 p.

REZENDE, M. Avaliação da inserção do fisioterapeuta na Saúde da Família de Macaé/RJ: a contribuição deste profissional para a acessibilidade da população idosa às ações de saúde das equipes. Um estudo de caso. Dissertação (Mestrado). Escola Nacional de Saúde Pública Sérgio Arouca/Ensp. Rio de Janeiro, 2007.

RIBEIRO, K. S. Q. A atuação da Fisioterapia na Atenção Primária à Saúde. Fisioterapia Brasil, v. 3, n. 5, p. 311318, São Paulo, 2002.

A contribuição da extensão comunitária para a formação acadêmica em fisioterapia. Fisioterapia e Pesquisa, v. 12, n. 3, São Paulo, 2005.

RODRIGUES, Adriano Conrado. Reabilitação: práticas inclusivas e estratégias para a ação: tecnologias de atenção, tecnologia assistiva: fisioterapia, fonoaudiologia, terapia ocupacional. São Paulo: Andreoli, 2008. 
SILVA, D. J.; ROS, M. A. da. Inserção de profissionais de fisioterapia na equipe de saúde da família e Sistema Único de Saúde: desafios na formação. Ciência \& Saúde Coletiva, Rio de Janeiro, v. 12, n. 6, p. 1673-1681, 2007.

VASCONCELOS, E. M. Educação popular: de uma prática alternativa a uma estratégia de gestão participativa das políticas de saúde. Physis: Revista de Saúde Coletiva, v. 14, n. 1, Rio de Janeiro, jan./jun. 2004.

WORLD HEALTH ORGANIZATION. Declaration of AlmaAta. International Conference on Primary Health Care. Alma-Ata: World Health Organization, 1978. 